\title{
Composición y fluctuación estacional de especies formadoras de florecimientos macroalgales en la laguna costera El Carmen, Cárdenas, Tabasco, golfo de México
}

\section{Seasonal composition and fluctuation of bloom forming macroalgae in the coastal lagoon El Carmen, Cárdenas, Tabasco, Gulf of Mexico}

\author{
Diego Armando Falcón-Vidal ${ }^{1}$, Ma. Guadalupe Rivas-Acuña ${ }^{2}$ y Alejandra Piñón-Gimate ${ }^{3 *}$
}

\begin{abstract}
RESUMEN
La presencia, distribución y abundancia de macroalgas están relacionadas con variaciones de los parámetros físicos y químicos; el incremento en su biomasa se vincula al enriquecimiento, por nutrientes, de las zonas costeras, por actividades antropogénicas. Los florecimientos macroalgales están conformados por especies de macroalgas efímeras de rápido crecimiento. En el presente trabajo, se describen los cambios en la composición y biomasa de florecimientos macroalgales en una laguna costera. Durante 3 épocas del año, lluvias (octubre de 2015), nortes (febrero de 2016) y secas (julio de 2016), se midieron variables fisicoquímicas en 5 sitios de la laguna El Carmen, Cárdenas, Tabasco; también, biomasa macroalgal (método de transectoscuadrantes). Las diferencias en las variables fisicoquímicas y la biomasa, entre épocas y sitios, se estimaron mediante análisis de varianza; igualmente, se realizó un análisis de componentes principales (ACP). Se obtuvieron 7 especies importantes. La mayor biomasa total correspondió a Gracilaria blodgetti (4225.1 g) seguida de Acanthophora spicifera (794.6 g). Los florecimientos macroalgales se encontraron solamente en el sitio 1 y en el 5, y se observó que, dependiendo de la época y el sitio, varió la composición de especies y la biomasa. El ACP mostró que la biomasa macroalgal está relacionada con las variables fisicoquímicas, dependiendo de la especie. En la región, las actividades antropogénicas que se realizan alrededor de las lagunas costeras impactan a estas últimas, por lo que este tipo de estudios es de importancia en el monitoreo de la laguna. Este es el primer estudio sobre florecimientos macroalgales en la región.
\end{abstract}

Palabras clave: macroalgas, cambios estacionales, biomasa, nutrientes, análisis de componentes principales.

1 Posgrado en Ciencias Ambientales, División Académica de Ciencias Biológicas, Universidad Juárez Autónoma de Tabasco, Avenida Universidad s/n, Colonia Magisterial, Villahermosa, Centro, Tabasco 86040, México.

2 División Académica de Ciencias Biológicas, Universidad Juárez Autónoma de Tabasco, Avenida Universidad s/n, Colonia Magisterial, Villahermosa, Centro, Tabasco 86040, México.

3 Instituto Politécnico Nacional-Centro Interdisciplinario de Ciencias Marinas (CICIMAR-IPN), Laboratorio de Macroalgas, Avenida Politécnico Nacional s/n, Colonia Playa Palo de Santa Rita, La Paz, Baja California Sur 23090, México. apinong@ipn.mx* 


\section{ABSTRACT}

The presence, distribution, and abundance of macroalgae are related to the fluctuations of physical and chemical parameters; the increase in macroalgae biomass is associated with nutrient enrichment of the coastal zones derived from anthropogenic activities. Macroalgae blooms are usually comprised of one or more ephemeral macroalgae species with rapid growth. In the present study, changes in the composition and biomass of macroalgae blooms of a coastal lagoon are described. Physicochemical variables were measured in 5 sites of El Carmen lagoon, Cárdenas, Tabasco in an annual cycle during 3 seasons, rainy (October, 2015), north winds (February, 2016), and dry (July, 2016). Macroalgae biomass was also measured using a transect-quadrat method. Differences in variables and biomass between seasons and sites were estimated with an ANOVA. In addition, a Principal Component Analysis (PCA) was conducted in order to observe the relations between environmental variables and biomass. A total of 7 species were found to be important. The highest total biomass corresponded to G. blodgetti $(4225.1 \mathrm{~g})$, followed by A. spicifera (794.6 g). Macroalgae blooms were found only in sites 1 and 5, and their species composition and biomass varied depending on site and season. The PCA showed that macroalgae biomass was related to physicochemical variables depending on the species. In the region, the anthropogenic activities surrounding the lagoons heavily affect them; therefore, this type of study is important to monitor the lagoon. This is the first study of macroalgae blooms in the region.

Keywords: macroalgae, seasonal changes, biomass, nutrients, Principal Component Analysis.

\section{INTRODUCCIÓN}

Las macroalgas son organismos fotosintéticos que enriquecen con oxígeno las aguas poco profundas, son uno de los principales componentes del bentos de las zonas costeras y participan en el reciclaje del carbono y nitrógeno. Su presencia, abundancia y distribución responden a las fluctuaciones estacionales de los parámetros hidrográficos tales como temperatura, salinidad y nutrientes (N y P) (Darley, 1982; Josselyn \& West, 1985; Kentula \& De Witt, 2003), así como a las interacciones biológicas de los sistemas (Neushul \& Coon, 1971; Darley, 1982).

Alrededor del mundo, la población humana se asienta en las cerca- nías de las lagunas costeras, ideales para desarrollar actividades económicas tales como la pesca, la acuicultura y el turismo. Adicionalmente, se llevan a cabo actividades en las cuencas de drenaje, como la agricultura, la ganadería y la industria. Sin embargo, estas llevan al enriquecimiento de nutrientes y tanto la aparición como el desenvolvimiento de florecimientos macroalgales, por consiguiente (Piñón-Gimate et al. 2008)

En México, existen algunos estudios que relacionan la abundancia de macroalgas con la presencia de nutrientes. Águila-Ramírez et al. (2005) reportaron cambios en la biomasa promedio de Ulva. OchoaIzaguirre (1999) encontró en el estero 
de Urías (Mazatlán, Sinaloa) que Ulva lactuca y Gracilaria sjoestedtii fueron las macroalgas más abundantes, mientras que Enteromorpha clathrata (actualmente Ulva clathrata) se presentó cerca del estero del Infiernillo, donde fue la más cuantiosa.

En la misma área, Ochoa-Izaguirre et al. (2002) registraron valores elevados de nutrientes $(14.8 \mu \mathrm{g} \mathrm{N}$ y $1.0 \mu \mathrm{M}$ de $\mathrm{P}$ disuelto), durante la época seca y la de lluvias (12.4 $\mu \mathrm{g} \mathrm{N}$ y 1.7 $\mu \mathrm{M}$ de fósforo disuelto), con los cuales se asoció la presencia del género Ulva. Magallanes-Beltrán (2002) señaló que en la Bahía de Altata, en la época de secas, la mayor cobertura de macroalgas correspondió a diferentes especies de Ulva. Chávez-Sánchez (2012) indicó que para la Bahía de La Paz las especies más abundantes fueron Caulerpa verticillata y Acanthophora spicifera, cuya cantidad estuvo relacionada con los nutrientes.

En cuanto al golfo de México, en particular en el estado de Tabasco, los estudios sobre macroalgas son pocos y se refieren a listados ficoflorísticos. Por ejemplo, Orozco-Vega \& Dreckmann (1995) reportaron para la laguna de Mecoacán 6 especies de algas rojas y un alga parda; Ramírez (1996), 24 especies de Rhodophyta (8 familias y 14 géneros); Dreckmann \& De Lara-Isassi (2000), a Gracilaria caudata para la laguna de Mecoacán. Quiroz-González et al. (2017) y Quiroz-González et al. (2018) informaron, en total, so- bre 75 especies para el estado. En la laguna El Carmen, Cárdenas, se tiene el trabajo de Pérez-Jiménez (2017), quien hizo la revisión ficológica de las algas de la laguna. No obstante, en la región no existe ningún estudio que dé a conocer cómo es la biomasa de las macroalgas en sistemas costeros del estado y si hay alguna relación de esta con las concentraciones de nutrientes. Por ello, el objetivo de este estudio fue determinar los cambios en la composición y biomasa estacional de especies formadoras de florecimientos macroalgales de la laguna costera El Carmen, Cárdenas, Tabasco, México, y la asociación de estas con variables fisicoquímicas y biológicas.

\section{MATERIALES Y MÉTODOS}

\section{Área de estudio}

La laguna El Carmen está ubicada al suroeste del golfo de México. Tiene un área de $92 \mathrm{~km}^{2}$, por lo que, al considerarse su extensión, se planteó realizar recolectas sistemáticas 3 veces al año, durante las épocas marcadas en el estado (nortes, secas y lluvias), en puntos que incluyeran los diferentes ambientes bentónicos que se presentan (Gutiérrez-Estrada \& Galaviz-Solís, 1983; Bello et al. 2009) y en donde se ha observado presencia de macroalgas (obs. pers.). Las localidades seleccionadas se enlistan a continuación (Fig. 1). 


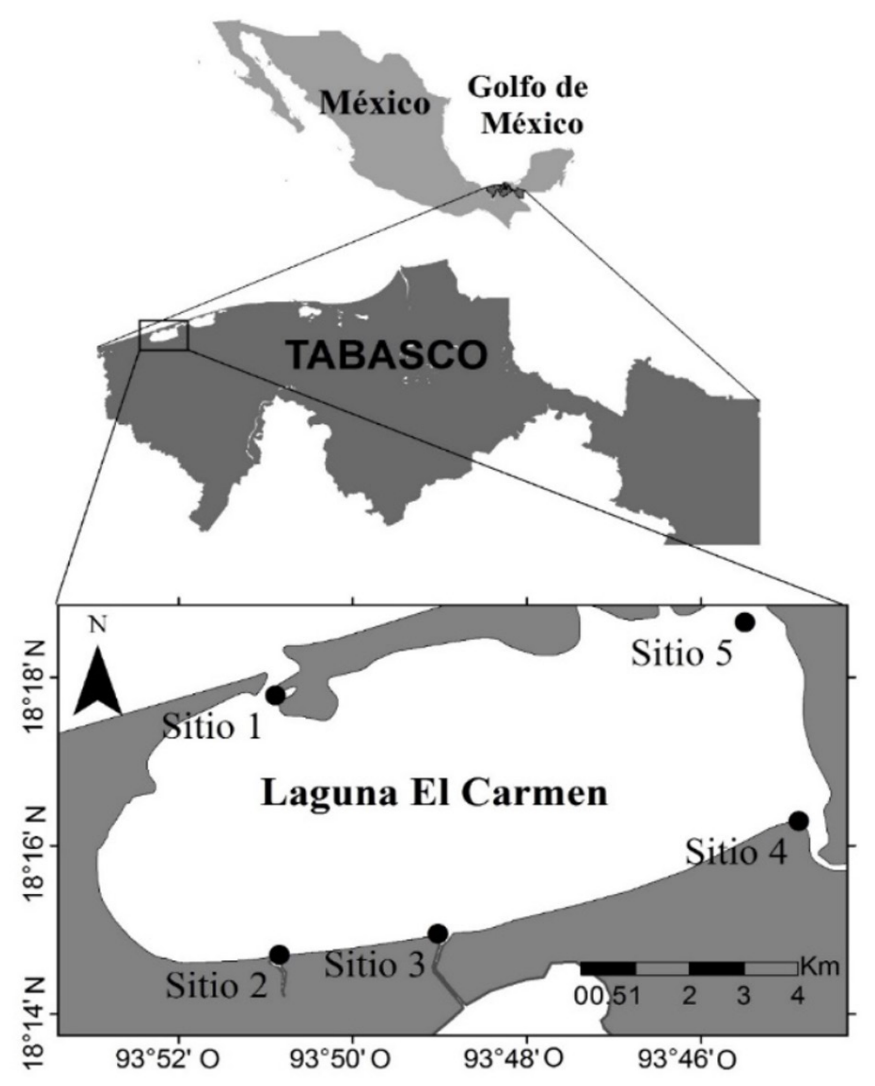

Fig. 1. Área de estudio, se indican los 5 sitios de muestreo en la laguna costera El Carmen, Cárdenas, Tabasco, México

Fig. 1. Study area in El Carmen lagoon, Cárdenas, Tabasco, Mexico, showing the 5 sampling sites

1) Boca de Santa Ana: Es el punto ubicado más al norte del estado y de la zona de muestreo. Es el que tiene las mayores características marinas de la laguna, se caracteriza por tener entrada constante de agua marina con salinidades de hasta 37 UPS. El sustrato es prin- cipalmente arenoso, aunque existen áreas con praderas de pastos marinos (Thalassia testudinum y Halodule wrightii), secciones de manglar, bancos de ostiones y fragmentos con escolleras.

2) Boca del arroyo El Zapote: Este sitio se caracteriza por tener 
sustrato arenoso-limoso, con presencia de manglares. Recibe aportes de agua dulce provenientes del arroyo El Zapote, por lo que la salinidad es variable en relación con el flujo y reflujo de la marea.

3) Boca de la laguna La Palma: Al igual que el sitio anterior, el sustrato es principalmente arenoso-limoso, con zonas de manglar y escurrimientos de agua dulce de la laguna La Palma.

4) Desembocadura del río San Felipe: En este sitio desemboca el río San Felipe en la laguna costera. Se encuentran, como sustrato, bancos de ostión, manglares, maderos colocados artificialmente y arena.

5) Boca de la laguna Pajonal: Este sitio está conformado, principalmente, por bancos de ostión, en los primeros $30 \mathrm{~m}$; posteriormente, fondo limoso, y por manglares. Se ubica en los límites entre la laguna El Carmen y la laguna Pajonal. Al igual que el sitio 1, presenta salinidades altas de hasta 34 UPS.

\section{Recolecta de muestras de agua y me-} dición de parámetros ambientales

Las estaciones de muestreo fueron georreferenciadas con un GPS (Garmin). La temperatura se midió con un termómetro de mercurio $\left(0-100^{\circ} \mathrm{C}\right)$, la salinidad se conoció con un refractómetro (Atago 0-100 PSU 8808), el oxígeno disuelto se obtuvo empleando una sonda YSI 540 y la transparencia del agua, con un disco de Secchi.

Para la determinación de los nutrientes (nitratos, nitritos, nitrógeno amoniacal y ortofosfatos), se recolectaron muestras de $1 \mathrm{~L}$ de agua durante la baja mar, a una profundidad de $0.50 \mathrm{~m}$, desechando las primeras 3 muestras y quedándonos con la cuarta. Las muestras se almacenaron en neveras con hielo y se trasladaron al Laboratorio de Aguas y Suelo de la Coordinación de Vinculación y Servicios (COVINSE) de la Universidad Juárez Autónoma de Tabasco (UJAT), para su análisis.

\section{Análisis de nutrientes}

El nitrito se determinó a partir del método de diazotización, aplicado al agua de mar; los nitratos, con base en la reducción a nitrito por cadmio; el amonio, partiendo de la óxido-reducción azul de indofenol. La estimación de la concentración de fosfato en el agua de mar se logró con la formación de un complejo de fosfomolibdato y su posterior reducción, con un compuesto azul que se lee en el espectrofotómetro, una longitud de onda de $885 \mathrm{~nm}$ (Strickland \& Parsons, 1972).

\section{Muestreo de macroalgas}

Durante un ciclo anual, se realizaron recolectas en octubre de 2015, así como en febrero y julio de 2016, épocas que correspondían a lluvias, nortes y secas, respectivamente. Un florecimiento macroalgal fue considerado como aquel en donde la 
presencia de macroalgas fuera conspicua, cubriera una extensión de al menos $50 \mathrm{~m}$ paralelos a la línea de costa y en donde cada especie que tuviera una biomasa de mínimo $1 \mathrm{gr} \mathrm{m}^{-2}$ fue considerada importante y formadora del florecimiento (Piñón-Gimate et al. 2008). La biomasa macroalgal se estimó mediante un muestreo sistemático en la zona submareal (Downing \& Anderson, 1985); en cada florecimiento, se colocaron 5 transectos de $30 \mathrm{~m}$ perpendiculares a la línea de costa. Cada uno estuvo dividido en 5 puntos equidistantes entre sí, en los cuales se recolectaron las macroalgas contenidas en un cuadrante de $1 \mathrm{~m}^{2}$ (25 muestras), por buceo libre (Ochoa-Izaguirre, 1999). Las algas fueron separadas por morfotipo, etiquetadas y guardadas en bolsas de plástico individuales para transportarlas al laboratorio.

\section{Determinación de la biomasa ma- croalgal}

Las algas recolectadas y separadas se lavaron con agua dulce, para remover el excedente de sedimentos y epibiontes. Se colocaron sobre papel absorbente, con miras a eliminar el exceso de agua y, posteriormente, fueron pesadas con una balanza electrónica con precisión de $\pm 1 \mathrm{~g}$ (Ohaus, Cseries). Solamente las especies importantes fueron consideradas para este estudio. La biomasa total se consideró como la sumatoria de cada especie trascendental (de 1 a
7 especies sobresalientes) en los 3 sitios, en las 3 épocas y se expresó en $\mathrm{g}$ (en peso húmedo). La biomasa total estacional se estimó como la sumatoria de cada especie importante ( 1 a $X$ especies importantes) en los 3 sitios, en una de las 3 épocas y se expresó en $g$ (en peso húmedo). La biomasa de las especies preponderantes por sitio de colecta y época del año es el promedio del peso de cada especie, en un lugar y en un periodo del año; se logró calculando la sumatoria del peso $(\mathrm{g})$ de la especie y dividiéndola entre el número total de cuadrantes de $1 \mathrm{~m}^{2}(25)$, por lo que la biomasa se expresó en $\mathrm{g} \mathrm{m}^{-2}$ (peso húmedo) (Piñón-Gimate et al. 2008).

\section{Identificación taxonómica de las macroalgas}

Para la identificación taxonómica de las macroalgas consideradas importantes, se tomó una submuestra que se fijó en formaldehído al 4\% con agua de mar; el análisis de las muestras se realizó en los laboratorios de Docencia de la División Académica de Ciencias Biológicas de la UJAT y en el Herbario de la Facultad de Ciencias de la Universidad Nacional Autónoma de México (UNAM).

Los ejemplares se identificaron a partir de su morfología externa, interna y reproductiva, con un estereoscopio (Olympus SZ2-IL-ST, Tokio, Japón y ZEIGEN ZEZEZ2100). Se hicieron preparaciones y cortes histológicos que fueron fijados con 
gelatina glicerinada al $75 \%$ y teñidos con cristal violeta para su observación en un microscopio óptico (Leica DM500 LAS EZ, Alemania) (Pérez-Jiménez, 2017). Se determinó el material ficológico mediante el uso de claves hasta el nivel taxonómico más bajo posible (Earle, 1972; Littler et al. 1989; Littler \& Littler, 2000; de la Garza, 2003). Para la actualización nomenclatural y sistemática, se utilizó AlgaeBase (Guiry \& Guiry, 2017; Pérez-Jiménez, 2017).

\section{Análisis estadístico}

Con el fin de observar si existían diferencias significativas en la biomasa de las especies entre épocas, sitios y variables ambientales, se realizaron análisis de varianza (ANDEVA) de una vía $(\alpha=0.05)$.

Posteriormente, se llevó a cabo un análisis de componentes principales (ACP) utilizando la biomasa de las macroalgas (variable dependiente), más los valores promedios de nutrientes, temperatura y salinidad (variables independientes), con el objetivo de identificar la posible correlación entre las variables ambientales y la biomasa de las macroalgas importantes (Santa María-Del Ángel et al. 1992). Los análisis se ejecutaron con ayuda del software STATISTICA 8.0 (Statsoft, 2007).

\section{RESULTADOS}

\section{Parámetros hidrográficos}

Se observaron algunas variaciones en los parámetros hidrográficos medidos, así como en las concentraciones de los nutrientes en la columna de agua, dependiendo del sitio y de la época del año. Se notó que la temperatura mostró un patrón estacional, en la época de nortes, en el sitio 1 . Se registró la temperatura más baja en $24^{\circ} \mathrm{C}$ y el valor más alto en la temporada de secas, en el sitio, fue $230.4^{\circ} \mathrm{C}$ (Fig. 2a).

La salinidad mostró una clara variabilidad entre épocas y sitios muestreados $(\mathrm{F}(2,12)=2.5738, P>$ $0.05)$. En la época de nortes, en el sitio 3, se observó el valor más bajo (10 UPS), mientras que, en la misma temporada, en el sitio 1, se obtuvo el más alto (35 UPS). A excepción del sitio 1, en la temporada de secas es cuando se presentaron los valores mayores de salinidad, mientras que en la temporada de nortes se obtuvieron valores más bajos, salvo en los sitios 1 y 2 (Fig. 2b).

Respecto al oxígeno disuelto, se observaron diferencias significativas entre sitios y épocas (F $(2,12)$ $=112.5130, P>0.05)$, con los valores más bajos en la época de lluvias, a excepción del sitio $2\left(0.14 \mathrm{mg}^{-1}\right)$, y el valor máximo en la temporada de nortes, en el 3 (13.9 $\mathrm{mg} \mathrm{l}^{-1}$ ) (Fig. 2c). La transparencia de la columna de agua presentó variaciones entre sitios 
y épocas $((\mathrm{F}(2,12)=7.59, P<0.05)$ y su rango estuvo entre 20 y $110 \mathrm{~cm}$ (Fig. 2d).
Se observó que la concentración de $\mathrm{NO}_{2}$ presentó pocas variaciones en el tiempo y el espacio $(\mathrm{F}(2,12)=2.02$,
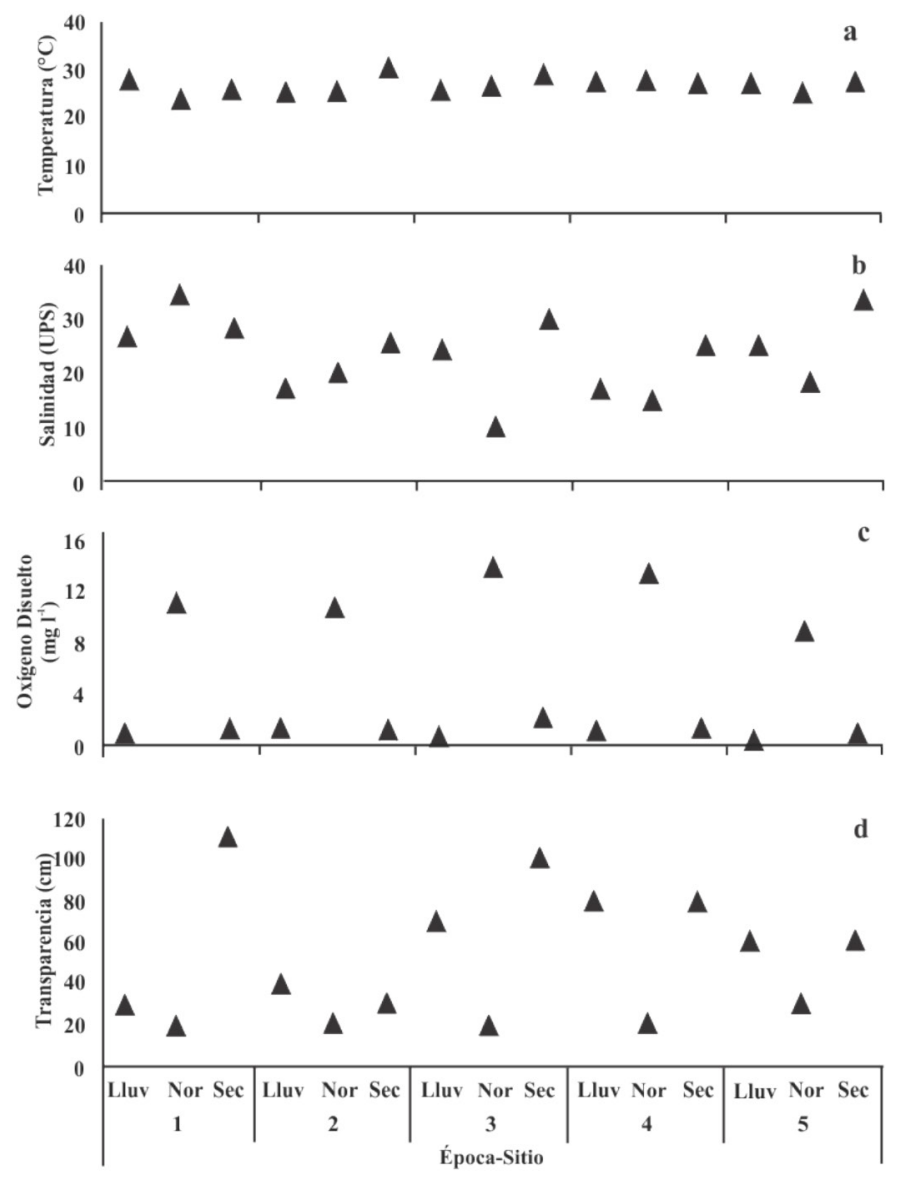

Fig. 2. Parámetros físicos por sitio y época (Lluv $=1$ luvia, Nor $=$ nortes y $\mathrm{Sec}=$ secas). a) Temperatura, b) salinidad, c) oxígeno disuelto y d) transparencia de la laguna El Carmen, Cárdenas, Tabasco, México, durante el ciclo anual octubre de 2015 a julio de 2016

Fig. 2. Values of the physical parameters for each site and season (Lluv = rainy, Nor $=$ north, and $\mathrm{Sec}=$ dry). a) Temperature, b) salinity, c) dissolved oxygen and d) transparency of El Carmen lagoon, Cárdenas, Tabasco, Mexico, during the annual cycle from October 2015 to July 2016 
$P>0.05)$. En la época de lluvias, se re- la temporada de nortes, en el sitio 2 gistró el valor más alto correspondiente $\quad(0.0002 \mu \mathrm{M})($ Fig. 3a).

al sitio $3(0.010 \mu \mathrm{M})$ y el más bajo en
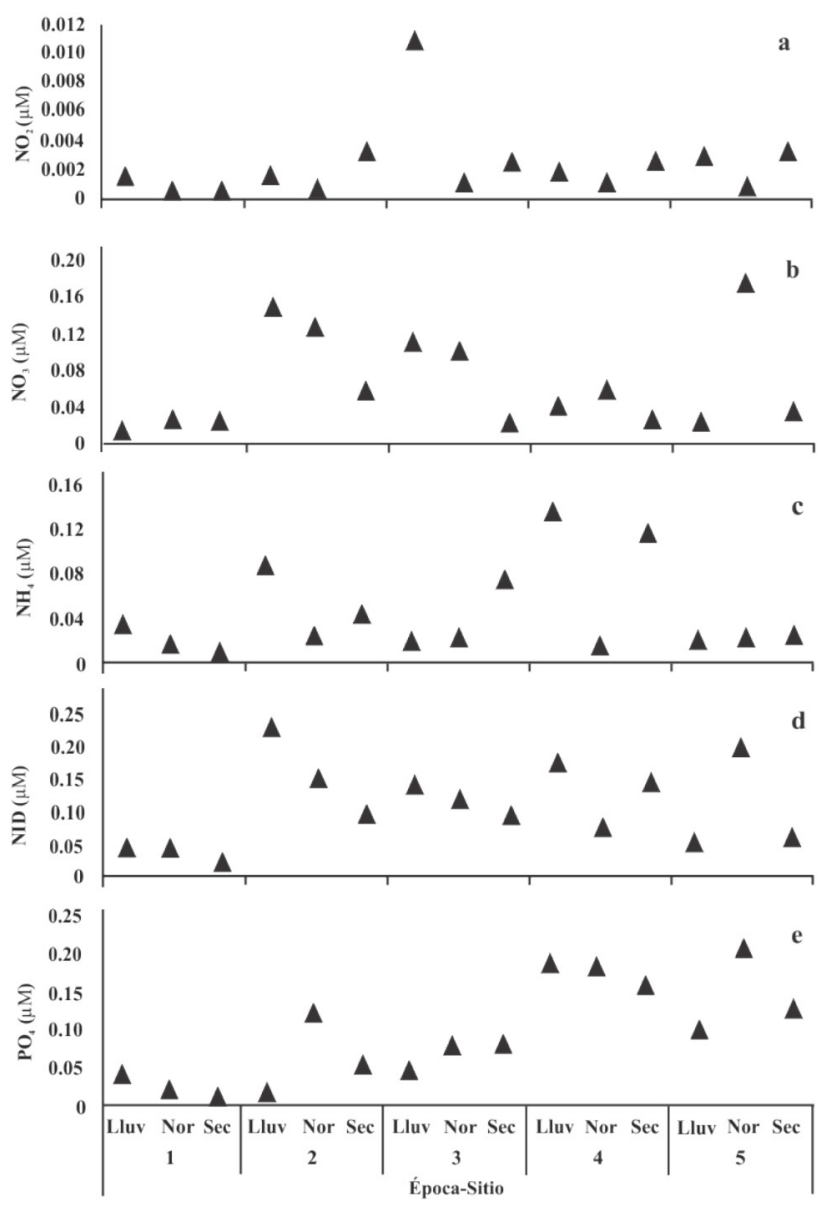

Fig. 3. Valores promedio de la concentración de nutrientes por sitio y época $($ Lluv $=$ lluvia, Nor $=$ nortes y $\mathrm{Sec}=$ secas $)$. a) Nitritatos, b) nitritos, c) amonia, d) NID y e) ortofosfatos de la laguna El Carmen, Cárdenas, Tabasco, México, durante el ciclo anual octubre de 2015 a julio de 2016

Fig. 3. Average values of nutrient concentration per site and season (Lluv = rainy, Nor $=$ north, and $\mathrm{Sec}=$ dry). a) nitrates, b) nitrite, c) ammonium, d) NID, and e) orthophosphates of El Carmen lagoon, Cárdenas, Tabasco, Mexico, during the annual cycle from October 2015 to July 2016 
El mayor valor del $\mathrm{NO}_{3}(\mathrm{~F}(2,12)$ $=2.38, P>0.05)$ se observó en la época de nortes, en el sitio $5(0.17 \mu \mathrm{M})$ (Fig. 3b). Se vio que la mayor concentración de $\mathrm{NH}_{4}(\mathrm{~F}(2,12)=2.38, P>$ $0.05)$ fue en la época de lluvias, en el sitio 4 (0.13 $\mu \mathrm{M})$ (Fig. 3c). En cuanto al nitrógeno inorgánico disuelto (NID), no se encontraron diferencias significativas entre sitios ni entre épocas $(\mathrm{F}(2,12)=2.09, P>0.05)$, por lo que el valor máximo de la temporada de lluvias se presentó para el sitio 2 $(0.23 \mu \mathrm{M})$ (Fig. 3d).

$\mathrm{El} \mathrm{PO}_{4}$ dejó ver variación significativa entre sitios $(\mathrm{F}(2,12)=8.7431, P$ $<0.05)$ pero no entre épocas: el mayor valor se obtuvo en la época de nortes, en el sitio $5(0.20 \mu \mathrm{M})$ (Fig. 3e).

\section{Biomasa macroalgal}

Se identificó un total de 7 especies importantes. En el sitio 1, se encontraron A. spicifera, G. blodgetti,
Gracilaria tikvahiae y Rosenvingea intricata (Cuadro 1), pertenecientes a la División Rhodophyta, mientras que en el sitio 5 se hallaron 4 especies Chondria littoralis, G. tikvahiae (División Rhodophyta), Dictyota sp. y Grateloupia filicina (División Ochrophyta-Phaeophyta) (Cuadro 1). En los sitios 2 y 3, no se observó presencia de macroalgas en ninguna de las épocas; en el 4, se vieron algunas especies, sin embargo, no fue considerado como florecimiento macroalgal ni las especies fueron tomadas en cuenta como importantes. Se obtuvo una biomasa total de 6374.3 g de macroalgas importantes, de la cual 1804.6 g correspondió a la época de lluvias, $3715.3 \mathrm{~g}$ a la de nortes y 854.4 g para secas. La especie con la mayor biomasa fue G. blodgetti y la de menor, G. filicina.

Cuadro 1. Biomasa total (g) de las especies importantes formadoras de florecimientos macroalgales en la laguna costera El Carmen, Cárdenas, Tabasco, México. Se indica su presencia por sitio

Table 1. Total biomass of important species of macroalgae blooms in El Carmen lagoon, Cárdenas, Tabasco, Mexico. Presence is indicated at each site

\begin{tabular}{lccc}
\hline \multirow{2}{*}{ Especie } & Biomasa total & \multicolumn{2}{c}{ Sitio } \\
\cline { 3 - 4 } & $(\mathbf{g})$ & Sitio 1 & Sitio 2 \\
\hline Gracilaria blodgetti Harvey & 4225.1 & $\sqrt{ }$ & \\
Acanthophora spicifera (M. Vahl) Børgesen & 794.6 & $\sqrt{ }$ \\
Rosenvingea intricata (J. Agardh) Børgesen & 712.4 & $\sqrt{ }$ & \\
Gracilaria. tikvahiae McLachlan & 472.2 & & $\sqrt{ }$ \\
Chondria littoralis Harvey & 350.7 & $\sqrt{ }$ & $\sqrt{ }$ \\
Dictyota sp. & 128.3 & $\sqrt{ }$ & $\sqrt{ }$ \\
Grateloupia filicina (J. V. Lamouroux) C.Agardh & 55.7 & $\sqrt{ }$ & \\
\hline
\end{tabular}


Se observó que la biomasa biomasa promedio en la época de macroalgal varió dependiendo del lluvias $\left(26.7 \pm 33.0 \mathrm{~g} \mathrm{~m}^{-2}\right)$, mientras sitio y en las 3 épocas estudiadas, que G. blodgetti fue más abundante en tanto en la composición de especies los periodos de nortes y secas, con una como en la biomasa. En el sitio 1, el biomasa de $138.7 \pm 100$ y $5.0 \pm 1.3 \mathrm{~g}$ alga roja A. spicifera fue la de mayor $\mathrm{m}^{-2}$, respectivamente (Fig. 4a).
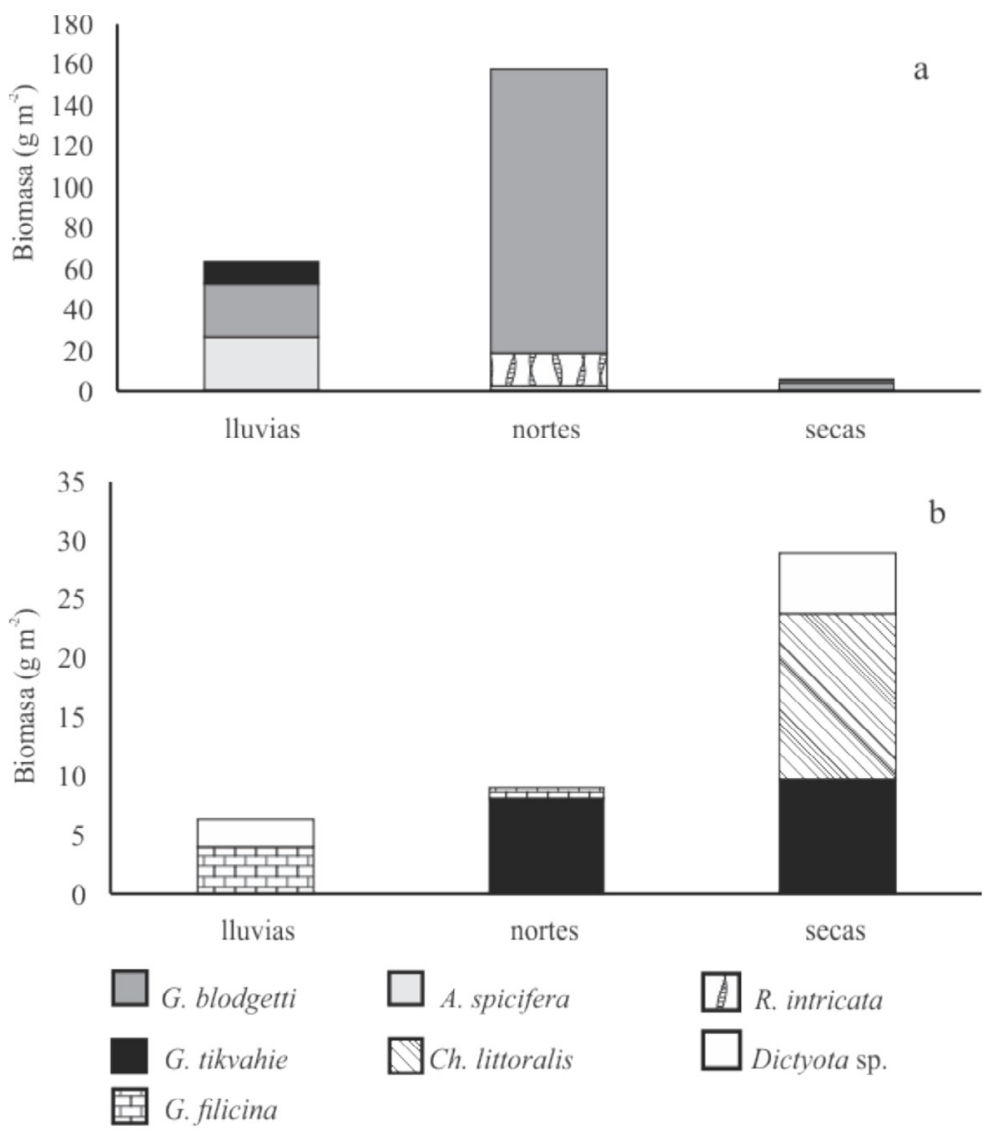

Fig. 4. Biomasa promedio por sitio de muestreo y época del año de la laguna El Carmen, Cárdenas, Tabasco, México, durante el ciclo anual octubre de 2015 a julio de 2016. Se indican las especies correspondientes a cada barra. a) $=$ sitio 1, b) $=$ sitio 5 Fig. 4. Biomass average values per site and season of El Carmen lagoon, Cardenas, Tabasco, Mexico, during the annual cycle from October 2015 to July 2016. Species corresponding to each bar are indicated. a) $=$ site $1, b)=$ site 5 
En el sitio 5, el alga roja G. filicina fue la que tuvo la mayor biomasa en la época de lluvias $\left(4 \pm 3.4 \mathrm{~g} \mathrm{~m}^{-2}\right)$, mientras que $G$. tikvahiae mostró la mayor biomasa en la temporada de nortes y secas $(8.0 \pm 7.2$ y $9.7 \pm 11.6 \mathrm{~g}$ $\mathrm{m}^{-2}$, respectivamente) (Fig. $4 \mathrm{~b}$ ).

\section{Relación de la biomasa con las va- riables hidrográficas}

En el análisis de componentes principales (ACP), se incluyeron temperatura, salinidad, $\mathrm{NO}_{2}, \mathrm{NO}_{3}, \mathrm{NH}_{4}$, $\mathrm{NID}, \mathrm{PO}_{4}$ y biomasa de las especies importantes. Los resultados mostraron que 3 de los factores obtenidos tuvieron un eigenvalor mayor a 1 ; sin embargo, las relaciones se explican con base en los factores 1 y 2 que dan el $63.7 \%$ de la variación.

De acuerdo con la carga de cada componente, se observó que la mayor variabilidad del primer factor correspondió, principalmente, a la temperatura, los nutrientes $\left(\mathrm{NH}_{4}\right.$ y $\left.\mathrm{NO}_{2}\right) \mathrm{y}$, en menor medida, a la salinidad. Para el segundo factor, la mayor variabilidad se presentó con base en $\mathrm{PO}_{4}$ y al $\mathrm{NO}_{2}$ $\mathrm{y}$, en menor escala, en la temperatura. Se observaron relaciones de la biomasa de algunas especies importantes con las variables ambientales. En el primer factor, la biomasa de A. spicifera, $G$. blodgetti y $R$. intrincata se encontró correlacionada positivamente con la temperatura y presentó una correlación negativa con el $\mathrm{NO}_{3}, \mathrm{NID}$ y $\mathrm{PO}_{4}$. En el segundo factor, observamos que la biomasa de G. filicina, G. tikvahiae y Dictyota sp. estuvo correlacionada positivamente con la concentración de $\mathrm{PO}_{4}$ y negativamente correlacionada con la salinidad (Cuadro 2, Fig. 5).

Cuadro 2. Correlación del análisis de componentes principales de las variables ambientales y la biomasa de las macroalgas importantes. A s: A. spicifera, $\mathrm{G}$ b: G. blodgetti, $\mathrm{G}$ t: G. tikvahiae, G f: G. filicina, D c: D. cervicornis, $\mathrm{R}$ i: $R$. intrincata. Letras negras indican correlación

Table 2. Correlation of the Principal Component Analysis of the environmental variables and the biomass of important macroalgae species. A s: A. spicifera, $\mathrm{G}$ b: $G$. blodgetti, $\mathrm{G}$ t: G. tikvahiae, $\mathrm{G}$ f: G. filicina, D c: Dictyota sp., R i: R. intrincata. Numbers in bold correspond to correlations

\begin{tabular}{lcc}
\hline \multicolumn{1}{c}{ Variable } & Factor 1 & Factor 2 \\
\hline $\mathrm{NO}_{2}$ & 0.45 & 0.55 \\
$\mathrm{NO}_{3}$ & $\mathbf{- 0 . 6 8}$ & 0.3 \\
$\mathrm{NH}_{4}$ & $\mathbf{0 . 6}$ & 0.33 \\
$\mathrm{NID}$ & -0.57 & 0.35 \\
$\mathrm{PO}_{4}$ & -0.44 & $\mathbf{0 . 6 3}$ \\
$\mathrm{Temp}$ & $\mathbf{0 . 8 3}$ & 0.14 \\
$\mathrm{Sal}$ & 0.18 & $\mathbf{- 0 . 5 8}$ \\
$\mathrm{A} \mathrm{s}$ & $\mathbf{0 . 8 3}$ & -0.23 \\
$\mathrm{G} \mathrm{b}$ & $\mathbf{0 . 8 3}$ & -0.25 \\
$\mathrm{G} \mathrm{t}$ & $\mathbf{0 . 7 3}$ & $\mathbf{0 . 6 2}$ \\
$\mathrm{G} \mathrm{f}$ & 0.23 & $\mathbf{0 . 8 3}$ \\
$\mathrm{D} \mathrm{sp}$ & 0.23 & $\mathbf{0 . 8 3}$ \\
$\mathrm{R} \mathrm{i}$ & $\mathbf{0 . 8 3}$ & -0.25 \\
\hline
\end{tabular}




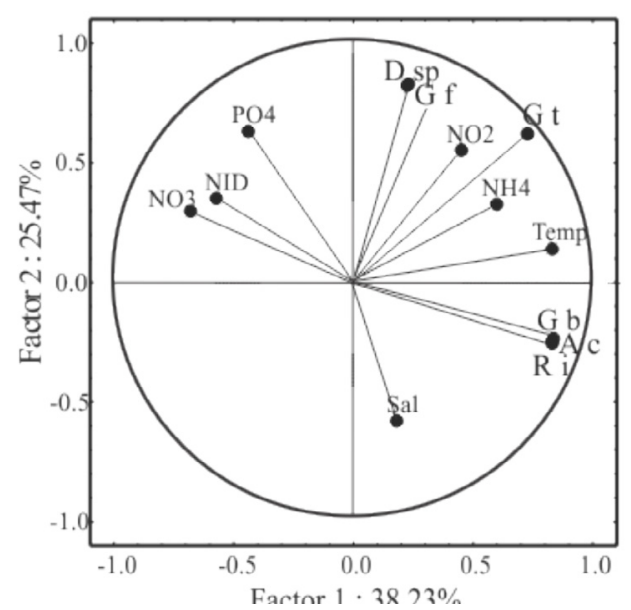

Fig. 5. Análisis de componentes principales mostrando la correlación de las variables ambientales y la biomasa de macroalgas. A s: $A$. spicifera, $\mathrm{G}$ b: G. blodgetti, $\mathrm{G}$ t: G. tikvahiae, G f: G. filicina, D c: Dictyota sp., R i: R. intrincata

Fig. 5. Principal Component Analysis showing the correlation between environmental variables and macroalgae biomass. A s: A. spicifera, $\mathrm{G}$ b: G. blodgetti, G t: G. tikvahiae, $\mathrm{G}$ f: G. filicina, D c: Dictyota sp., R i: $R$. intrincata

\section{DISCUSIÓN}

Una característica importante del comportamiento ambiental de las lagunas costeras en latitudes tropicales es la heterogeneidad espacial, que depende de los aportes continentales, marinos y del clima, que influyen en la precipitación y evaporación, las cuales contribuyen a los cambios en la concentración salina y de nutrientes de las masas de agua (De la Lanza-Espino et al. 1998). Los patrones de temperatura y salinidad se comportaron acordes con la época del año, coincidiendo con lo reportado en otros estudios llevados a cabo en la región (De la Lanza-Espino et al. 1998; De la Lanza-Espino \& GómezAguirre, 1999). Las concentraciones de nutrientes encontradas en este estudio son menores que las reportadas para otras lagunas costeras, por ejemplo, aquellas del golfo de California, ya que registran concentraciones promedio para el NID de hasta $51 \mu \mathrm{M}$ y de hasta $4 \mu \mathrm{M}$ promedio para fósforo disuelto (Piñón-Gimate et al. 2008), muy por arriba de los encontrados en este estudio. Aun así, se ha reportado que las concentraciones de nutrientes en lagunas costeras del golfo de México pueden ser muy variables (MuciñoMarquez et al. 2017).

Se encontraron 7 especies importantes formadoras de florecimientos macroalgales, lo cual ha sido observado alrededor del mundo desde hace ya algunas décadas, en donde una o varias especies dominan el florecimiento por sobre las otras (e. g. Valiela et al. 1997; Whitehouse \& Lapointe, 2015). La biomasa macroalgal ha sido utilizada como un indicador en estudios de calidad del agua (Bricker et al. 2003; Lapointe et al. 2015), ya que esta se incrementa en respuesta al enriquecimiento de nutrientes que son generalmente de origen antropogénico (Sfriso \& Marcomini, 
1997; Valiela et al. 1997). Sin embargo, también se han observado florecimientos macroalgales de especies del género Ulva, en sitios con enriquecimientos por surgencias de tipo natural (Pacheco-Ruiz et al. 2002).

En los florecimientos de la laguna costera El Carmen, las especies $G$. blodgetti y A. spicifera fueron las de mayores biomasas acumuladas, 4225 y $794.6 \mathrm{~g}, \mathrm{y}$, en promedio, 138.7 y 26.7 $\mathrm{g} \mathrm{m}^{-2}$, respectivamente. Esto es similar a estudios en otras localidades de México, en los cuales se ha observado que 1 o 2 especies de macroalgas dominan los florecimientos macroalgales (e. g. Piñón-Gimate et al. 2008, Chávez-Sánchez, 2012). En lagunas costeras de Sinaloa, la especie roja Gracilaria vermiculophylla presentó biomasas altas de entre 640 y $2910 \mathrm{~g} \mathrm{~m}^{-2}$, que, mediante un análisis de componentes principales, se observaron asociadas al enriquecimiento de nutrientes, principalmente fósforo, de tipo antropogénico, puesto que, en esta región, las descargas de nutrientes provienen de efluentes de aguas residuales, de la agricultura y de granjas camaronícolas, primordialmente (Piñón-Gimate et al. 2008). Recientemente, en el estado de Sonora, se llevó a cabo un estudio en el cual la biomasa macroalgal fue tomada como un indicador de calidad del agua; G. lemaneiformis fue la especie dominante con biomasas acumuladas de hasta 20 ton en un sitio, con influencia de aguas de drenaje prove- nientes de la ciudad cercana, por lo que se concluyó que la biomasa estaba relacionada con las aguas ricas en nutrientes de este efluente (Ruiz-Ruiz et al. 2017).

Chávez-Sánchez(2012)encontró que la biomasa de G. vermiculophylla en la Bahía de La Paz fue de $11 \mathrm{~g} \mathrm{~m}^{-2}$ y esta se vinculó con la temperatura, mientras que el mismo autor halló que la biomasa de A. spicifera, la especie con mayor biomasa $\left(149 \mathrm{~g} \mathrm{~m}^{-2}\right)$, estuvo relacionada con las concentraciones altas de nitrógeno total determinado. En la misma bahía, también se reportó $A$. spicifera en otro sitio y se encontró que su presencia está enlazada con ciclos estacionales (Gabrielle-Schnoller, 2011). La especie $A$. spicifera ha sido registrada como invasora en otras regiones del mundo, por ejemplo, en las costas de Hawaii se encontró cubriendo grandes extensiones de arrecifes coralinos. Esta especie fue introducida y comenzó a formar grandes florecimientos macroalgales que se han asociado al enriquecimiento por aguas residuales, aunque no se han hecho estudios en particular al respecto (O’Doherty \& Sherwood, 2007). Las biomasas encontradas en el presente estudio para las especies G. blodgetti y $A$. spicifera fueron menores que las reportadas en sitios con alta influencia de actividades tales como agricultura y camaronicultura, y mayores que aquellas donde hay poco desarrollo alrededor de la zona costera. 
En este trabajo, también se reportaron como especies importantes G. filicina, Dictyota sp., $R$. intrincata y C. littoralis, las cuales no habían sido registradas previamente como formadoras de florecimientos. Sin embargo, se ha observado en otros estudios que las especies que dominan los florecimientos son aquellas con características morfológicas que les permiten una rápida absorción de nutrientes $\mathrm{y}$, por lo tanto, responden rápidamente al enriquecimiento con biomasas elevadas (Lotze \& Schramm, 2000).

Se observó que las especies de macroalgas que dominaron los florecimientos variaron de una época a otra, así como su biomasa; esto ha sido reportado en otras indagaciones. Por ejemplo, Ochoa-Izaguirre et al. (2002) observaron un cambio en la composición de especies del estero de Urias en Sinaloa, México, dependiendo de la época del año. Tales autores describieron un cambio, ya que, durante la época de secas, las macroalgas dominantes, conspicuas en términos cualitativos, fueron $U$. lactuca, $U$. lobata y E. clathrata, mientras que en la temporada de lluvias, en el mismo estero, las especies dominantes fueron Gracilariopsis sjoestedii y Caloglossa leprieurii. Así mismo, en otras lagunas costeras del estado, se observó un cambio en la composición de las especies de los florecimientos, que se asociaron a la estacionalidad propia de cada especie que proliferara cuando las condiciones para su crecimiento son óptimas (Piñón-Gimate et al. 2008). En la Bahía de La Paz, se observó que la composición de especies del mismo género Ulva también variaba dependiendo de la época del año, así como notaron variación dependiendo del sitio (Chávez-Sánchez et al. 2018). La composición de macroalgas varía aun dentro de la misma laguna, estero o bahía, dependiendo del sitio de colecta y las actividades que se desarrollen alrededor (OchoaIzaguirre et al. 2002; Piñón-Gimate et al. 2008; Chávez-Sánchez et al. 2018). Lo encontrado en esta indagación coincide con lo reportado, ya que se observó un cambio en la composición del florecimiento, dependiendo de la época del año. Una de las especies presenta la mayor biomasa, por ejemplo, en el sitio 1, G. blodgetti pasó de una menor biomasa en la época de lluvias a una mayor en la de nortes y poca biomasa en la de secas; $R$. intricata solo estuvo presente en la temporada de nortes; mientras que, en el sitio 5, las especies importantes fueron $G$. tikvahie, encontrada en la época de nortes y secas, y G. filicina, que estuvo presente en lluvias y nortes, mas desapareció en el lapso de secas.

En el presente estudio, el incremento de la biomasa de las macroalgas se relacionó con el enriquecimiento de nutrientes mediante el análisis multivariante. Aunque las concentraciones 
de nutrientes son menores que las reportadas para otros sitios con presencia de florecimientos macroalgales, se ha notificado que aun en zonas oligotróficas se pueden dichos florecimientos, pero que podrían ser dominados por más especies (Valiela et al. 1997). En este caso, los nutrientes provenientes de las descargas de aguas residuales y de desechos domésticos de la Villa Andrés Sánchez Magallanes, en el sitio 5, así como los escurrimientos agrícolas procedentes de los ríos y arroyos que descargan al sistema lagunar y desechos de las granjas camaronícolas y de ostión en esta zona de la laguna estarían favoreciendo la biomasa de las macroalgas. En otros trabajos, se ha observado que las especies del género Gracilaria presentan un crecimiento relacionado con el incremento de los nutrientes al sistema (Friedlander \& Dawes, 1985). Por ejemplo, G. tikvahiae junto con Hypnea musciformis han formado florecimientos macroalgales en la Indian River Lagoon, Florida, que han estado asociados con el enriquecimiento de nutrientes. Estas especies pertenecientes a la División Rhodophyta, por su morfología están más adaptadas a desarrollarse en ambientes en donde las concentraciones de nutrientes son variables; estos se almacenan efectivamente cuando cuentan con pulsos altos (Lapointe, 1981; Whitehouse \& Lapointe, 2015). Esto pudiera estar sucediendo en el sitio 5, donde la biomasa de Gracilaria fue la más alta, viéndose favorecida por la presencia de nutrientes de origen antropogénico. Igual en el sitio 1 , donde G. blodgetti es la de mayor biomasa, que también pudiera estar beneficiada por las actividades que se desarrollan en este sitio, como los bancos de ostión.

La salinidad pareció estar relacionada negativamente con la abundancia de G. tikvahiae, G. filicina y Dictyota sp., lo cual ha sido observado en lagunas costeras de Sinaloa para especies del género Gracilaria (Piñón-Gimate, 2008). Aun cuando las concentraciones de nutrientes parecieran no ser limitantes, otros factores pueden serlo, ya que los cambios en la salinidad podrían limitar a las especies que no se ven favorecidas por salinidades bajas. Por ejemplo, para A. spicifera se llevó a cabo un estudio que demostró que esta se puede desarrollar adecuadamente en salinidades desde 25 hasta 40UPS, pero debajo de ellas o por arriba sufre afectaciones en su biomasa y en su coloración (Pereira et al. 2017). Esto pudiera explicar la presencia/ausencia de estas especies importantes en los otros sitios visitados, además del tipo de sustrato que se encuentra en ellos, ya que 2, 3 y 4 no presentan un sustrato en el cual las macroalgas se puedan adherir, mientras que en 1 y 5 las macroalgas se hallaron adheridas a los restos de conchas y estructuras que sobresalían sobre el 
sustrato de limo que predomina en la laguna (obs. pers.).

La presencia de florecimientos macroalgales estuvo relacionada con los nutrientes que en esta laguna costera se derivan de las actividades antropogénicas; sin embargo, su existencia se ve limitada por las características de cada sitio, tal como el sustrato y la salinidad, así como la ecología propia de cada especie. El presente estudio es la base del conocimiento sobre los florecimientos macroalgales del golfo de México y la región, al igual que amplía el conocimiento sobre la presencia de aquellos en los litorales mexicanos. En otras investigaciones, se han llevado a cabo análisis moleculares, para confirmar las especies formadoras de florecimientos macroalgales, por lo que se recomienda que se hagan cuando sea posible. Es de importancia continuar con el monitoreo de estos florecimientos macroalgales, debido a que las zonas costeras brindan múltiples servicios ecosistémicos y los cambios en ellos pueden tener consecuencias que podrían causar una alteración en el medio.

\section{AGRADECIMIENTOS}

Al Proyecto Riqueza de macroalgas de la laguna El Carmen, Cárdenas, Tabasco, a cargo de Guadalupe Rivas-Acuña, del Programa del Fomento a la Investigación (PFI). A
Graciela María Pérez-Jiménez y Kiara Lizbeth Arévalo-Pérez por su ayuda en campo. A la beca CONACYT, otorgada para estudios de maestría a Diego Falcón-Vidal. A la beca Instituto Politécnico Nacional-EDI, concedida a Alejandra Piñón-Gimate. Al editor y a 2 revisores anónimos que han enriquecido este trabajo con sus valiosos y atinados comentarios.

\section{BIBLIOGRAFÍA}

Águila-Ramírez, R. N., Casas-Valdez, M. M., Hernández-Guerrero C. J. \& Marín-Álvarez, A. (2005). Biomass of Ulva spp. (Chlorophyta) in three locations along the bay front of La Paz, Baja California Sur, Mexico. Rev. Biol. Mar. Oceanogr., 40(1), 55-61. https://doi.org/10.4067/ S0718-19572005000100006

Bello, J., Cervantes-Abrego, M., Gómez, L., Magaña, V., Graizbzod, B. \& Hipolito-Rodríguez, P. (2009). Sitio piloto Sistema Lagunar Carmen-Pajonal-Machona. En J. Buenfil-Friedman (Ed), Adaptación a los impactos del cambio climático en los humedales costeros del Golfo de México (pp. 480-502). México: SEMARNAT.

Bricker, S. B., Ferreira, J. G. \& Simas, T. (2003). An integrated methodology for assessment of estuarine trophic status. Ecol. Mod., 169, 39-60. https://doi. org/10.1016/S0304-3800(03)00199-6.

Chávez-Sánchez, T. (2012). Composición y abundancia de especies de florecimientos macroalgales y su relación con variables ambientales en la Ensenada de La Paz, Baja California Sur. Tesis de Maestría no publicada, CIBNOR, La Paz, Baja California Sur. 
Chávez-Sánchez, T., Piñón-Gimate, A., Serviere-Zaragoza, E., López-Bautista, J. M. \& Casas-Valdez, M. (2018). Ulva blooms in the southwestern Gulf of California: Reproduction and biomass. Est. Coast. Shelf Sci., 200, 202-211. https://doi.org/10.1016/j. ecss.2017.11.007

Darley, W. M. (1982). Algal Biology. A physiological approach. Oxford, England: Backwell Scientific Publications.

De la Garza, A. (2003). Clave genérica de las algas rojas marinas macroscópicas y comunes de las costas de Veracruz, México. Tesis de licenciatura no publicada, Facultad de Ciencias, Universidad Nacional Autónoma de México.

De la Lanza-Espino, G., Santillán, N. S. \& Herrera, A. E. (1998). Análisis temporal y espacial fisicoquímico de una laguna tropical a través del análisis multivariado. Hidrobiol., 8, 89-96.

De la Lanza-Espino, G. \& Gómez-Aguirre, S. (1999). Fisicoquímica del agua y cosecha de fitoplancton en una laguna costera tropical. CIENCIA ergo-sum, 6 , 147-153.

Downing, J. A. \& Anderson, M. R. (1985). Estimating the standing biomass of aquatic macrophytes. Can. J. Fish. Aquat. Sci., 42, 1860-1869. https://doi. org/10.1139/f85-234

Dreckmann, K. M. \& De Lara-Isassi, G. (2000). Gracilaria caudata J. Agardh (Gracilariaceae, Rhodophyta) en el Atlántico mexicano. Hidrobiol., 10, 125130.

Earle, S. A. (1972). Benthic algae and seagrass species in the Gulf of México. In V. C. Bushnell (Ed.), Serial Atlas of the Marine Environment (pp. 25-29). Nueva York, EE. UU.: American Geographical Society.

Friedlander, M. \& Dawes, C. J. (1985). In situ uptake kinetic of ammonium and phosphate and chemical composition of the red seaweed Gracilaria tikvahiae. J. Phycol., 21, 448-453. https://doi.org/10.1111/j.0022-3646.1985.00448.x

Gabrielle-Schnoller, V. G. (2011). Variación espacial y datos reproductivos de Acanthophora spicifera (Ceramiales: Rhodophyta) en la playa Punta Roca Caimancito de la Bahía de La Paz, Baja California Sur, México. Tesis de Licenciatura no publicada. Universidad Autónoma de Baja California Sur, La Paz, Baja California Sur, México.

Guiry, M. \& Guiry, G. (2017). AlgaeBase. Recuperado en enero 31, 2017, disponible en http:// www.algaebase.org

Gutiérrez-Estrada, M. \& Galaviz-Solís, A. (1983). Morfología y sedimentos recientes de las lagunas El Carmen, $\mathrm{Pa}-$ jonal y La Machona, Tabasco. México. An. Inst. Cienc. Mar. Limnol., 10(1), 249-270.

Josselyn, M. N. \& West, J. A. (1985). The distribution and temporal dynamics on the estuarine macroalgal community of San Francisco Bay. Hydrobiol., 129, 139-152. https://doi.org/10.1007/ BF00048692

Kentula, M. E. \& De Witt, T. H. (2003). Abundance of seagrass (Zostera marina L.) and macroalgae in relation to the salinity-temperature gradient in Yaquina Bay, Oregon, USA. Estuaries, 26, 1130-1141. https://doi.org/10.1007/ BF02803369

Lapointe, B. E. (1981). The effects of light and nitrogen on growth, pigment content, and biochemical composition of Gracilaria foliifera v. Augustissima (Gigartinales, Rhodophyta). J. Phycol., 17, 90-95.

Lapointe, B. E., Herren, L. W., Debortoli, D. D. \& Vogel, M. A. (2015). Evidence 
of sewage-driven eutrophication and harmful algal blooms in Florida's Indian River Lagoon. Harmful Algae, 43, 82-102. https://doi.org/10.1016/j. hal.2015.01.004

Littler, D., Littler, M., Bucher, K. \& Norris, J. (1989). Marine plants of the Caribbean, a field guide from Florida to Brazil. Washington, D. C., EE. UU.: Smithsonian Institution Press.

Littler, D. \& Littler, M. (2000). Caribbean Reff Plants. Washington, D. C., EE. UU.: OFFSHORE GRAPHICS, INC.

Lotze, H. K. \& Schramm, W. (2000). Ecophysiological traits explain species dominance patterns in macroalgal blooms. J. Phycol., 36, 287-295. https://doi.org/10.1046/j.1529-8817.2000.99109.x

Magallanes-Beltrán, J. (2002). Las macroalgas de Sinaloa: un recurso olvidado. En J. Cifuente-Lemus \& J. Gaxiola-López (Eds.), Atlas de la Biodiversidad de Sinaloa (pp. 43-48). Culiacán, Sinaloa, México: El Colegio de Sinaloa.

Muciño-Márquez, R. E., Aguirre-León, A. \& Figueroa-Torres, M. G. (2017). Evaluación del estado trófico en los sistemas fluvio-lagunares Pom-Atasta y Palizada del Este, Campeche, México. Hidrobiológica, 27(3), 281-291.

Neushul, M. D. \& Coon D. (1971). Bibliography on the ecology and taxonomy of marine algae. En J. Parker (Ed.), Select Papers in Phycology (pp. 12-17) Nebraska, Nebraska, EE. UU.: University of Nebraska.

Ochoa-Izaguirre, M. J. (1999). Composición y distribución de macroalgas en el sistema lagunas de Urías (Mazatlán, Sinaloa, México): Variación estacional de la biomasa en relación con la disponibilidad de nutrientes y algunos factores ambientales. Tesis de Maestría no publicada. ICMyL, UNAM, Mazatlán, Sinaloa, México.
Ochoa-Izaguirre, M. J., Carballo, J. \& PáezOsuna, F. (2002). Qualitative changes in macroalgal assamblages under two contrasting climatic conditions in a subtropical estuary. Bot. Mar., 45, 130-138. https://doi.org/10.1515/ BOT.2002.014

O'Doherty, D. C. \& Sherwood, A. R. (2007). Genetic Population Structure of the Hawaiian Alien Invasive Seaweed Acanthophora spicifera (Rhodophyta) as Revealed by DNA Sequencing and ISSR Analyses. Pac. Sci., 61(2), 223-233. https://doi.org/10.2984/1534-6188(2007)61[223:GPSOTH]2.0.CO;2

Orozco-Vega, H. \& Dreckmann, K. M. (1995). Macroalgas estuarinas del litoral mexicano del Golfo de México. Criptogamie, Algologie., 16(3), 189198.

Pacheco-Ruíz, I. J., Zertuche-González, A., Chee-Barragán, A. \& Arroyo-Ortega. E. (2002). Biomass and potential commercial utilization of Ulva lactuca (Chlorophyta, Ulvaceae) beds along the north-west coast of the Gulf of California. Phycologia, 41(2), 199-201. https://doi.org/10.2216/i0031-888441-2-199.1

Pereira, D. T., Simioni, C., Filipin, E. P., Bouvie, F., Ramlov, F., Maraschin, M. \& Schmidt, É. C. (2017). Effects of salinity on the physiology of the red macroalga, Acanthophora spicifera (Rhodophyta, Ceramiales). Act. Bot. Bras., 31, 555-565. https://doi. org/10.1590/0102-33062017abb0059

Pérez-Jiménez (2017). Macroalgas de la laguna el Carmen, Cárdenas, Tabasco, México. Tesis de licenciatura no publicada. Universidad Juárez Autónoma de Tabasco, Villahermosa, Tabasco, México. 
Piñón-Gimate, A. (2008). Caracterización de florecimientos macroalgales de lagunas costeras de Sinaloa y su relación con factores fisicoquímicos. Tesis de doctorado no publicada. Universidad Nacional Autónoma de México, Mazatlán, Sinaloa, México.

Piñón-Gimate, A., Serviere-Zaragoza, E., Ochoa-Izaguirre, M. J. \& Páez-Osuna, F. (2008). Species composition and seasonal changes in macroalgal blooms in lagoons along the southeastern Gulf of California. Bot. Mar., 51, 112-123. https://doi.org/10.1515/BOT.2008.013

Quiroz-González, N., León-Álvarez, D. \& Rivas-Acuña, M. (2017). Nuevos registros de algas verdes marinas (Ulvophyceae) para Tabasco, México. Act. Bot. Mex., 118, 121-138. https://doi. org/10.21829/abm118.2017.1204

Quiroz-González, N., León-Álvarez, D. \& Rivas-Acuña, M. (2018). Biodiversidad de algas rojas marinas (Rhodophyta) en Tabasco, México. Act. Bot. Mex., 123, 103-120. https://doi.org/10.21829/ abm123.2018.1253

Ramírez, A. (1996). Estudio preliminar de las algas rojas (Rhodophyta) del litoral del Estado de Tabasco, México. Tesis de licenciatura no publicada, Universidad Nacional Autónoma de México, Campus Iztacala.

Ruiz-Ruiz, T., Arreola-Lizarraga, J. A., Morquecho, L., Méndez-Rodríguez, L. C., Martínez-López, A. \& Mendoza-Salgado, R. (2017). Detecting Eutrophication Symptoms in a subtropical Semi-Arid Coastal Lagoon by Means of Three Different Methods. Wetlands., 37(6), 1105-1118. https://doi. org/10.1007/s13157-017-0944-y
Santa María-Del Ángel, E., Millán-Núñez, R. \& Cajal-Medrano, R. (1992). Efecto de la energía cinética turbulenta sobre la distribución espacial de la clorofila $a$ en una pequeña laguna costera. Cienc. Mar., 18, 1-16.

Sfriso, A. \& Marcomini, A. (1997). Macrophyte production in a shallow coastal lagoon. Part I: Coupling with chemico-physical parameters and nutrient concentrations in waters. Mar. Environ. Res., 44, 351-375. https://doi. org/10.1016/S0141-1136(97)00012-3

StatSoft. (2007). STATISTICA for Windows computer program, version 8.0. By StatSoft, Tulsa, OK.

Strickland, J. D. \& Parsons, T. R. (1972). A practical handbook of seawater analysis. Fisheries Research Board of Canada.

Valiela, I., McClelland, J., Hauxwell, J., Bher, P., Hersh, D. \& Formean, K. (1997). Macroalgal blooms in shallow estuaries: contols and ecophysiological and ecosystem consequences. Limnol. Oceanogr., 42, 1105-1118. https://doi. org/10.4319/lo.1997.42.5 part_2.1105

Whitehouse, L. N. \& Lapointe, B. E. (2015). Comparative ecophysiology of bloom-forming macroalgae in the Indian River Lagoon, Florida: Ulva lactuca, Hypnea musciformis, and Gracilaria tikvahiae. J. Exp. Mar. Biol. Ecol., 471, 208-216. https://doi.org/10.1016/j.jembe.2015.06.012 